\title{
Catalytically active bovine serum amine oxidase bound to fluorescent and magnetically drivable nanoparticles
}

This article was published in the following Dove Press journal:

International Journal of Nanomedicine

2 May 2012

Number of times this article has been viewed

\section{Giulietta Sinigaglia' \\ Massimiliano Magro' \\ Giovanni Miotto' \\ Sara Cardillo' \\ Enzo Agostinelli ${ }^{2,3}$ \\ Radek Zboril ${ }^{4}$ \\ Eris Bidollari ${ }^{2,3}$ \\ Fabio Vianello'}

'Department of Biological Chemistry, University of Padua, Padua, Italy; ${ }^{2}$ Istituto Pasteur-Fondazione Cenci Bolognetti, Department of Biochemical Sciences "A. Rossi Fanelli”, SAPIENZA University of Rome, Rome, Italy; ${ }^{3} \mathrm{CNR}$, Institute Biology and Molecular Pathology, Rome, Italy; ${ }^{4}$ Regional Centre of Advanced Technologies and Materials, Department of Physical Chemistry, Palacky University, Olomouc, Czech Republic
Correspondence: Fabio Vianello Department of Comparative Biomedicine and Food Science, University of Padua Agripolis -Viale dell'Università 16 , Legnaro, 35020 (PD), Italy

$\mathrm{Tel}+390498276863$

$\mathrm{Fax}+390498073310$

Email fabio.vianello@unipd.it

\begin{abstract}
Novel superparamagnetic surface-active maghemite nanoparticles (SAMNs) characterized by a diameter of $10 \pm 2 \mathrm{~nm}$ were modified with bovine serum amine oxidase, which used rhodamine B isothiocyanate (RITC) adduct as a fluorescent spacer-arm. A fluorescent and magnetically drivable adduct comprised of bovine serum copper-containing amine oxidase (SAMN-RITC-BSAO) that immobilized on the surface of specifically functionalized magnetic nanoparticles was developed. The multifunctional nanomaterial was characterized using transmission electron microscopy, infrared spectroscopy, mass spectrometry, and activity measurements. The results of this study demonstrated that bare magnetic nanoparticles form stable colloidal suspensions in aqueous solutions. The maximum binding capacity of bovine serum amine oxidase was approximately $6.4 \mathrm{mg} \mathrm{g}^{-1}$ nanoparticles. The immobilization procedure reduced the catalytic activity of the native enzyme to $30 \% \pm 10 \%$ and the Michaelis constant was increased by a factor of 2 . We suggest that the SAMN-RITC-BSAO complex, characterized by a specific activity of $0.81 \mathrm{IU} \mathrm{g}^{-1}$, could be used in the presence of polyamines to create a fluorescent magnetically drivable $\mathrm{H}_{2} \mathrm{O}_{2}$ and aldehydes-producing system. Selective tumor cell destruction is suggested as a potential future application of this system.
\end{abstract}

Keywords: amine oxidase, hydrogen peroxide production, superparamagnetic nanoparticles, rhodamine isothiocyanate, fluorescent nanoparticles, fluorescent nanocatalyst

As a result of the rapid development of nanotechnology, magnetic nanoparticles are now widely studied. It has long been known that the physicochemical properties of magnetic nanoparticles can be vastly different from the properties of the corresponding bulk material. ${ }^{1}$

In comparison to micro- or submicrometric size particles, the advantage of using nanometric size particles is that they have a larger surface area that can be used to attach biomolecules. These biomolecules will enable the preparation of nanostructured biomaterial with a high loading per mass unit and the possibility of immobilizing biomolecules.

Magnetic separation is an interesting tool that is used in bioassays because, when compared to conventional chromatographic methods, the magnetic beads enable the isolation or extraction of a target molecule with higher efficiencies through the application of an external magnetic field. ${ }^{2-4}$ Due to the good biocompatibility and adequate functional groups for chemical fixation, magnetic nanoparticles modified on the surface by various recognition polymers can be used to immobilize specific biomolecules. Additionally, they are employed as a solid adsorbent and as magnetic separation of support from the reaction mixture. ${ }^{5}$ Magnetic nanoparticles are applicable 
in many areas such as the immobilization of proteins and enzymes, ${ }^{6}$ bioseparation, ${ }^{7}$ immunoassay, ${ }^{8}$ drug delivery, ${ }^{9}$ and biosensors. ${ }^{10}$

We have developed a new method to synthesize superparamagnetic nanoparticles constituted of stoichiometric maghemite $\left(\gamma-\mathrm{Fe}_{2} \mathrm{O}_{3}\right)$ with a dimension of approximately $10 \mathrm{~nm}$. These nanoparticles are characterized by specific chemical behavior without any superficial modification or coating derivatization. These bare iron nanoparticles are stable in water for several months as colloidal suspensions. They also present a high average magnetic moment and can be easily derivatized to immobilize specific organic molecules in a solution. ${ }^{11,12}$

The aim of this present work is to combine the advantages of immobilized proteins on nanomaterials and the easy operation of magnetic nanoparticles to develop an inexpensive and simple amine oxidase-based magnetic nanocatalyst tagged with a fluorescent probe.

Copper containing amine oxidases, $\mathrm{Cu}-\mathrm{AO}$ (EC 1.4.3.6), represents a heterogeneous class of enzymes (amine:oxygen oxidoreductase [deaminating] [copper-containing]) that is widely found in mammals, plants, and microorganisms. ${ }^{13}$ There has been an increase in research interest in this class of enzymes because they are involved in polyamine metabolism and in numerous physiopathological processes. ${ }^{14}$ A number of $\mathrm{Cu}-\mathrm{AOs}$ have been purified to homogeneity and characterized. These enzymes share some fundamental structural properties. They both are glycosylated homodimers measuring 70-95 kDa. Depending on their source, each monomer contains one tightly bound $\mathrm{Cu}$ (II) ion and one carbonyl-type group as cofactors. These cofactors are either 6-hydroxydopa quinone (2,4,5-trihydroxyphenylalaninequinone) or lysine tyrosylquinone. ${ }^{15,16}$

These enzymes operate by abstracting two electrons from aromatic and aliphatic primary amines and transferring them to molecular oxygen. The following equation demonstrates how this process produces the corresponding aldehyde, ammonia, and hydrogen peroxide:

$$
\begin{aligned}
& \text { R- }-\mathrm{CH}_{2}-\mathrm{NH}_{3}^{+}+\mathrm{O}_{2}+\mathrm{H}_{2} \mathrm{O} \stackrel{\text { Amine oxidase }}{\longrightarrow} \\
& \mathrm{R}-\mathrm{CHO}+\mathrm{NH}_{4}^{+}+\mathrm{H}_{2} \mathrm{O}_{2}
\end{aligned}
$$

Despite their wide distribution, the physiological role of $\mathrm{Cu}-\mathrm{AOs}$ is still unclear. Bovine serum amine oxidase (BSAO: EC 1.4.3.6) was used to generate cytotoxic polyamine metabolites. The oxidative deamination of spermine by BSAO generates $\mathrm{H}_{2} \mathrm{O}_{2}$, aldehydes, and ammonia. ${ }^{17-19}$ Hydrogen peroxide and aldehydes induce stress-activated signal transduction pathways, which leads to apoptotic and nonapoptotic cell death. ${ }^{20,21}$ Exposure of tumor cells to purified BSAO and spermine causes a timedependent decrease of cell viability ${ }^{21}$ and impairs the growth of mouse melanoma. ${ }^{22}$ Analogous to other radical forming processes, the formation of reactive oxygen species (ROS) and cytotoxic aldehydes from polyamines are currently being explored as a possible strategy in cancer therapy. ${ }^{23}$ Moreover, hydrogen peroxide and aldehydes generated by $\mathrm{BSAO/spermine} \mathrm{enzymatic} \mathrm{systems} \mathrm{have} \mathrm{been} \mathrm{shown}$ to overcome multidrug resistance (MDR) in human colon adenocarcinoma (LoVo) ${ }^{24,25}$ and melanoma (M14) cells. ${ }^{26}$ This is particularly interesting because one of the problems of conventional anticancer therapy is the development of drug resistance.

In the present work, we report a unique synthetic route of maghemite nanoparticles exhibiting excellent colloidal behavior without any additional organic or inorganic modification of their surface. These nanoparticles immobilized rhodamine B isothiocyanate (RITC), which acted as a fluorescent label, and a magnetically controllable rhodamine-based fluorescent nanocomposite has been synthesized as a result. Moreover, the rhodamine structure used in this research allows for the covalent attachment of various biomolecules. This has been demonstrated in the immobilization of BSAO. Acting as a monomolecular layer, the immobilized enzyme retains its catalytic activity towards oxidation of polyamines. The prepared fluorescent magnetically drivable nanocatalyst could be used as a nanodevice that selectively kills tumor cells by the in situ production of hydrogen peroxide and aldehydes. ${ }^{27}$ In order to achieve this potential clinical application, our system offers the dual advantage of being easily removed by nanomaterial superparamagnetism and of being easily detectable by fluorescence.

\section{Materials and methods Chemicals}

Chemicals were purchased at the highest commercially available purity and were used without further treatment. Iron(III) chloride hexahydrate (97\%), sodium borohydride $\left(\mathrm{NaBH}_{4}\right)$, RITC, rhodamine 110 , rhodamine B, fluorescein, fluorescein isothiocyanate (FITC), tetramethylammonium hydroxide, perchloric acid, and ammonia solution (35\% in water) were obtained from Aldrich (Sigma-Aldrich, Milan, Italy). Spermine tetrahydrochloride was obtained from Fluka (Buchs, Switzerland).

Amine oxidase from bovine serum was purified to electrophoretic homogeneity according to Turini et al. ${ }^{28}$ All of the 
samples that were used had a minimum specific activity on benzylamine oxidation of $0.38 \mathrm{IU} \mathrm{mg}^{-1}$. Assay was performed spectrophotometrically at $25^{\circ} \mathrm{C}$ by monitoring the formation of benzaldehyde at $250 \mathrm{~nm}$ absorbance $\left(\varepsilon=1.25 \times 10^{4} \mathrm{M}^{-1} \mathrm{~cm}^{-1}\right)$. International Unit (IU) is defined as $\mu$ moles of substrate oxidized per minute.

\section{Instrumentation}

Optical spectroscopy and fluorescence measurements were respectively performed in $1 \mathrm{~cm}$ quartz cuvettes using a Cary 50 spectrophotometer and a Cary Eclipse fluorescence spectrometer (Varian Inc, Palo Alto, CA).

Fourier transform-infrared (FTIR) spectra were acquired using a Thermo Nicolet NEXUS 670 FTIR instrument (Thermo Fisher Scientific, Waltham, MA). Nanoparticle samples were homogenized with $\mathrm{KBr}$ powder and pelletized using an 8-ton hydraulic press. Microscopic characterizations of the native maghemite particles, as well as rhodaminecoated particles, were performed using transmission electron microscopy (TEM). TEM micrographs were obtained using a Tecnai 12 microscope (FEI, Portland, OR) operating at $120 \mathrm{kV}$ with a point-to-point resolution of $1.9 \AA$.

\section{Synthesis of iron oxide nanoparticles}

A typical nanoparticle synthesis has already been described ${ }^{11,12}$ and can be summarized as follows: $\mathrm{FeCl}_{3} \times 6 \mathrm{H}_{2} \mathrm{O}(10.0 \mathrm{~g}$, $37 \mathrm{mmol})$ was dissolved in MilliQ grade water $(800 \mathrm{~mL})$ under vigorous stirring at room temperature. An $\mathrm{NaBH}_{4}$ solution $(2 \mathrm{~g}, 53 \mathrm{mmol})$ in ammonia $(3.5 \%, 100 \mathrm{~mL})$ was then quickly added to the mixture. Soon after the occurrence of the reduction reaction, the temperature of the system was increased to $100^{\circ} \mathrm{C}$ and kept constant for 2 hours. The material was cooled at room temperature and aged in water for another 12 hours. This product was separated by the imposition of an external magnet and washed several times with water. This material was transformed into a red-brown powder (final synthesis product) by drying and curing at $400^{\circ} \mathrm{C}$ for 2 hours. The resulting nanopowder showed a magnetic response upon exposure to a magnetic field. The final mass of the product was $2.0 \mathrm{~g}$ (12.5 mmol) of $\mathrm{Fe}_{2} \mathrm{O}_{3}$ and a yield of $68 \%$ was calculated.

The resulting nanoparticulated material was characterized by zero field and in field (5T) Mossbauer spectroscopy, FTIR spectroscopy, high-resolution TEM, X-ray powder diffraction, magnetization measurements, ${ }^{11,12}$ and constituted stoichiometric maghemite $\left(\gamma-\mathrm{Fe}_{2} \mathrm{O}_{3}\right)$ with a mean diameter of $10 \pm 2 \mathrm{~nm}$. Sonication in water (mod. 221, $48 \mathrm{kHz}, 50 \mathrm{~W}$; Branson Ultrasonic Corporation, Danbury, CT) can form a stable colloidal suspension without any organic or inorganic coverage. Notwithstanding, the surface of these bare maghemite nanoparticles shows peculiar binding properties and can be reversibly derivatized with selected organic molecules. These bare nanoparticles are called surface-active maghemite nanoparticles (SAMNs).

\section{Preparation of rhodamine bound nanoparticles}

The surface of bare SAMNs can be superficially and reversibly derivatized by simple incubation in $50 \mathrm{mM}$ tetramethylammonium perchlorate, $\mathrm{pH} 7.0$, in the presence of $50 \mu \mathrm{M}$ RITC. The amount of bound RITC was calculated from the disappearance of the RITC absorbance at $554 \mathrm{~nm}$ in the supernatant $\left(\varepsilon=6.6 \times 10^{4} \mathrm{M}^{-1} \mathrm{~cm}^{-1}\right)$. Following the application of an external magnet, SAMN-RITC suspensions were extensively washed in $50 \mathrm{mM}$ tetramethylammonium perchlorate and no leaching of the rhodamine derivative was evident, as determined by absorbance spectroscopy of the resulting supernatant upon external magnet application. The resulting fluorescent magnetic nanoparticles were comprised of $29.1 \pm 3.8 \mu \mathrm{mol}$ RITC per $\mathrm{g}$ of SAMNs.

\section{Preparation of bovine serum amine oxidase adduct with rhodamine-modified maghemite nanoparticles}

RITC can be used as a spacer-arm and presents the isothiocyanate functionality to which the primary amino groups of selected molecules can be covalently linked. In this way, a magnetically drivable fluorescent nanocarrier was prepared using the SAMN-RITC adduct. In the present work, the binding reaction was performed by adding $11 \mu \mathrm{M}$ BSAO in $0.1 \mathrm{M} \mathrm{KCl}$ containing $5 \mathrm{~g} \mathrm{~L}^{-1}$ SAMN-RITC nanoparticles and stirring overnight at $4^{\circ} \mathrm{C}$. The unbound enzyme was removed with the aid of an external magnet application using extensive nanoparticle washing.

\section{Determination of nanoparticle bound amine oxidase by mass spectrometry}

The nature and optical scattering properties of maghemite nanoparticles interferes with the determination of proteins bound on their surface by the classical protein determination methods. To overcome this limitation, an alternative approach based on protein quantitation by mass spectrometry was applied.

BSAO samples, native or nanoparticle bound, were extensively dialyzed against $40 \mathrm{mM}$ ammonium bicarbonate $(3 \times 2$ hour buffer changes with a 1000:1 volume ratio), 
dried under a vacuum, and resuspended in $100 \mu \mathrm{L}$ of $40 \mathrm{mM}$ ammonium bicarbonate. Native and SAMN-bound BSAO samples were normalized based on their enzymatic activity and incubated overnight at $37^{\circ} \mathrm{C}$ with sequencing grade trypsin (Promega, Madison, WI) and an enzyme to substrate ratio of 120 (w/w, based on specific activity of the native BSAO). After tryptic digestion, samples were centrifuged at $14000 \times g$ for 10 minutes and exposed to an external magnet for 30 minutes to remove SAMN. Finally, the supernatant were dried under a vacuum, dissolved in $20 \mu \mathrm{L}$ of $0.1 \%$ formic acid, and analyzed by liquid chromatography coupled with tandem mass spectrometry (LC-MS/MS) using a 6520 Q-TOF mass spectrometer, which was coupled on-line with a 1200 series high-pressure liquid chromatography system through a Chip Cube Interface (Agilent Technologies, CA). Each sample $(4 \mu \mathrm{L})$ was loaded onto a C18 large capacity chip-column that integrated a $160 \mathrm{~nL}$ capacity trap-column, an RP column $(75 \mu \mathrm{m} \times 150 \mathrm{~mm})$, connection capillaries, and a nanospray emitter. Solvent $\mathrm{A}$ was water/formic acid $0.1 \%$ and solvent $\mathrm{B}$ was acetonitrile/formic acid $0.1 \%$. Peptides were separated with a linear gradient of $0 \%-50 \%$ of solvent B in 50 minutes at a flow rate of $0.3 \mu \mathrm{L} \mathrm{min} \mathrm{m}^{-1}$. Mass spectra were acquired in a data-dependent mode; tandem mass spectra of the four most intense ions were acquired for each MS scan, which ranged from 350-3000 Da. The scan speed was set to $2 \mathrm{MS}$ spectra $\mathrm{s}^{-1}$ and $2 \mathrm{MS} / \mathrm{MS}$ spectra $\mathrm{s}^{-1}$. Capillary voltage was set to $1850 \mathrm{~V}$ and drying gas to $5 \mathrm{~L} \mathrm{~s}^{-1}$. Raw data files were converted into Mascot generic format (MGF) files using MassHunter Qualitative analysis software (Agilent Technologies, Santa Clara, CA). MGF files were analyzed using Mascot Search Engine server version 2.3 (Matrix Science, London, UK). Spectra were searched against the SwissProt database (May 2011 version, Taxonomy Mammalia, 65453 entries) with the following parameters: enzyme specificity was set to trypsin with up to two missed cleavages, and peptide and fragment tolerance were set to $6 \mathrm{ppm}$ and $0.05 \mathrm{Da}$, respectively. Oxidation of methionine was selected as a modification variable. Based on the search against the corresponding randomized database, false discovery rates (FDR) of $0.1 \%$ were calculated. Only identified peptides with a confidence of $\geq 99 \%$ were used to quantify the amount of BSAO bound to nanoparticles.

\section{Enzyme activity measurements}

A spectrophotometric assay was used to measure the activity of amine oxidase free in solution and immobilized on SAMNs. Initial rate measurements were carried out according to the peroxidase-coupled assay reported by Stevanato et al. ${ }^{29}$ The $\mathrm{H}_{2} \mathrm{O}_{2}$ produced during spermine oxidation was continuously monitored by the change of absorbance at $555 \mathrm{~nm}$, under continuous stirring, using an extinction coefficient, $\varepsilon$, of $2.2 \times 10^{4} \mathrm{M}^{-1} \mathrm{~cm}^{-1}$. Unless otherwise specified, all experiments were carried out in airsaturated solutions of 20 mM HEPES (4-(2-hydroxyethyl)-1piperazine-ethanesulfonic acid), $\mathrm{pH} 7.5$, at room temperature $\left(22^{\circ} \mathrm{C} \pm 1^{\circ} \mathrm{C}\right)$ in the presence of $0.1 \mathrm{mM}$ EDTA. Protein concentration was measured according to the Bradford method, ${ }^{30}$ which uses bovine serum albumin as a standard.

\section{Data analysis}

Catalytic constant $\left(\mathrm{k}_{\mathrm{c}}\right)$ and catalytic efficiency $\left(\mathrm{k}_{\mathrm{c}} / \mathrm{K}_{\mathrm{M}}\right)$ were calculated from the $\mathrm{V}_{\text {max }}$ and $\mathrm{K}_{\mathrm{M}}$ values and were obtained by nonlinear best fit of experimental data on MichaelisMenten equation and by assuming a BSAO molecular mass of $170 \mathrm{kDa}^{31}$

Experimental data were fitted using the Sigma Plot program (v. 10.0; Jandel Scientific, San Rafael, CA).

\section{Results Nanoparticles resuspension in different media}

In order to characterize the nanoparticle's surface, stable colloidal water suspensions are a necessity. In the present case, maghemite nanoparticles were synthesized according to an innovative method ${ }^{11,12}$ and suspended in bidistilled water using an ultrasonic bath (mod. 221 at $48 \mathrm{kHz}, 50 \mathrm{~W}$; Bransonic). Upon ultrasound application, suspensions were sampled and the absorbance was read every 30 minutes. It was found that, by measuring the increase of suspension absorbance during sonication, the dispersion exhibited exponential behavior. As previously described, the first order kinetic constants were in the range $10-100 \mathrm{mg} \mathrm{L}^{-1}$ and independent of the nanoparticles' concentration. ${ }^{12}$ In water, the dispersion kinetic constant resulted in $0.85 \pm 0.055 \mathrm{~h}^{-1}$. The colloidal suspension of maghemite nanoparticles was stable for more than 6 months without precipitation or aggregation phenomena.

Beside SAMN stability in water, the biotechnological applications of magnetic nanoparticles are supposed to be carried out in buffered solutions. According to previous research on the stabilizing/destabilizing effects of different cations and anions on iron oxide nanoparticle suspensions, ${ }^{32}$ the dispersion process of our bare SAMNs was carried out in $50 \mathrm{mM}$ tetramethylammonium perchlorate. Under these conditions, colloidal SAMN dispersions were stable and further experiments on the dispersion process of SAMN were performed using the same experimental conditions. 


\section{RITC binding to nanoparticle surface}

Preliminary experiments showed that the SAMN surface is able to selectively interact with different organic molecules and enzymes. For example, SAMNs are able to interact directly with avidin, but not with bovine serum amine oxidase. This suggests some kind of recognition process between SAMN surface and protein molecules, which will be explored elsewhere.

In order to improve our knowledge of maghemite nanoparticles' surface properties, various fluorescent dyes, characterized by similar structures and different functional groups, were tested (Figure 1).

Maghemite nanoparticles $\left(100 \mathrm{mg} \mathrm{L}^{-1}\right)$ were dispersed in $50 \mathrm{mM}$ tetramethylammonium perchlorate, $\mathrm{pH} 7.0$, and were incubated with the fluorescent probe $(10 \mu \mathrm{M})$. The test tube was gently shaken for one hour at room temperature. After the incubation period, nanoparticles were separated using an external magnetic field. The supernatant was checked at the maximum absorbance wavelength of the probe, as reported in Table $1 .{ }^{33}$

A significant fluorophore absorbance reduction was measured only when nanoparticles were incubated in the presence of RITC. No reduction of the absorbance was detected with other fluorescent probes. This suggests that only RITC is able to bind to the nanoparticle's surface. This indicates that the coexistence of isothiocyanate and an amino group on the molecule is necessary to anchor the molecule to the surface of the SAMN.
The binding phenomenon between maghemite nanoparticles and RITC is completed within a few minutes and forms a stable suspension of the fluorescent magnetic complex.

RITC binding on maghemite surface was determined as a function of fluorescent probe concentration, in the range $10-100 \mu \mathrm{M}$, in $50 \mathrm{mM}$ tetramethylammonium perchlorate, at $\mathrm{pH}$ 7. RITC concentrations above $100 \mu \mathrm{M}$ were not considered because the probe is not soluble above this concentration. In the concentration range explored, the amount of the bound probe on the nanoparticle's surface depended on its concentration in the solution. Experimental data were analyzed according to the Langmuir isotherm mode ${ }^{34}$ and the maximum-loading capacity, $\mathrm{q}_{\max }$, was $0.14 \mathrm{mg}$ RITC $\mathrm{mg}^{-1} \mathrm{SAMN}$. A Langmuir binding constant, $\mathrm{K}_{\mathrm{L}}$, of $61.7 \mathrm{~mL} \mathrm{mg}^{-1}$ was calculated. The result corresponded to 533 RITC molecules per nanoparticle.

After washing and freeze-drying, the adduct formed with maghemite nanoparticles and RITC (SAMN-RITC) was studied using FTIR spectroscopy. In Figure 2, the FTIR spectra of the SAMN-RITC complex, as well as that of the maghemite nanoparticle and the free RITC, are reported As shown in the figure, the comparison between the spectra of the free RITC and of the SAMN-RITC complex showed the relevant broadening of FTIR bands at $1412 \mathrm{~cm}^{-1}$, which is attributable to $\mathrm{CH}_{2}$ bending of $-\mathrm{N}-\left(\mathrm{CH}_{2}-\mathrm{CH}_{3}\right)_{2}$ groups, ${ }^{35}$ inset of Figure 2. Furthermore, the disappearance of the FTIR band at $2030-2150 \mathrm{~cm}^{-1}$ was observed and attributed to the isothiocyanate group. ${ }^{36}$ In contrast with the other probes<smiles>O=C(O)c1ccccc1-c1c2ccc(=O)cc-2oc2cc(O)ccc12</smiles>

Fluorescein

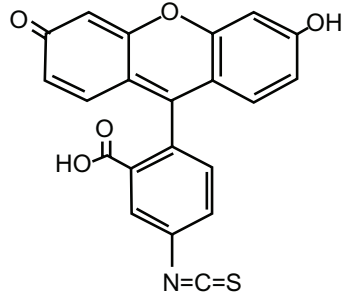

Fluorescein isothiocyanate

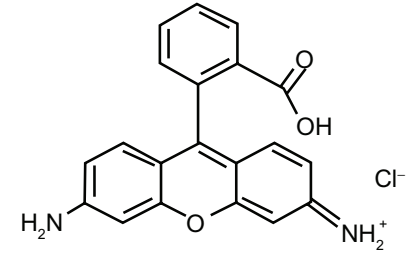

Rhodamine 110

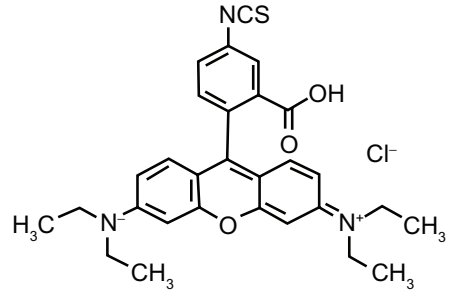

Rhodamine B

isothiocyanate

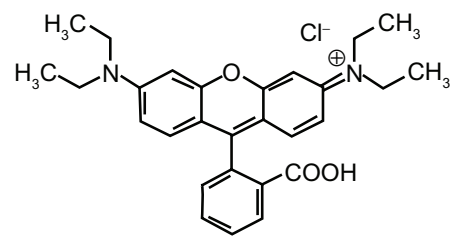

Rhodamine B

Figure I Structures of the fluorescent dyes used as probes for nanoparticle surface properties. 
Table I Optical characteristics of fluorescent dyes used to test maghemite nanoparticle surface properties

\begin{tabular}{|c|c|c|c|c|}
\hline Fluorescent probe & $\begin{array}{l}\text { Molar extinction } \\
\text { coefficient }\left(\mathrm{m}^{-1} \mathrm{~cm}^{-1}\right)\end{array}$ & $\begin{array}{l}\text { Excitation } \\
\text { wavelength }(\mathrm{nm})\end{array}$ & $\begin{array}{l}\text { Emission } \\
\text { wavelength }(\mathrm{nm})\end{array}$ & Quantum yield ${ }^{\mathrm{a}}$ \\
\hline Fluorescein & 65,000 & 494 & 520 & 0.79 \\
\hline Fluorescein isothiocyanate & 65,000 & 494 & 520 & 0.79 \\
\hline Rhodamine B & 106,000 & 554 & 580 & 0.49 \\
\hline Rhodamine $B$ isothiocyanate & 106,000 & 554 & 580 & 0.49 \\
\hline Rhodamine 110 & 90,000 & 498 & 520 & 0.9 \\
\hline
\end{tabular}

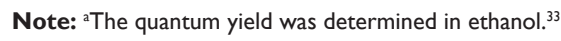

tested, this suggests the involvement of the $-\mathrm{N}=\mathrm{C}=\mathrm{S}$ moiety in the binding and confirms its responsibility in specific interaction with RITC.

SAMN-RITC was compared with free rhodamine and the fluorescence characteristics were determined. The SAMN-RITC complex is fluorescent and shows an excitation and emission spectra similar to unbound RITC. With respect to the free fluorophore, a quantum yield of $8.9 \% \pm 1.3 \%$ was estimated for the immobilized RITC, which is sufficient to apply the developed material as a magnetic fluorescent probe.

In order to improve our knowledge about the binding properties of RITC on SAMN's surface, it has been observed that the $\mathrm{pH}$ was fundamentally important in regulating the amount of adsorbed molecules. We have tested SAMN's capability in binding RITC in the $\mathrm{pH}$ range $2-10$ in $50 \mathrm{mM}$ tetramethylammonium perchlorate. The results in Figure 3 show that the phenomenon is well described by a sigmoidal behavior. This suggests the existence of two different binding modalities, in acidic and basic $\mathrm{pH}$ extremities, respectively. In particular, we observed that the ability of RITC to interact with nanoparticles increases at acidic $\mathrm{pH}$ values. The amount of bound RITC at $\mathrm{pH} 4.0$ was about 500 molecules per nanoparticle and the amount of bound RITC at basic $\mathrm{pH}$ values (pH 10.0) decreases to about 150 molecules per nanoparticle.

These data offer a new perspective about the behavior of RITC adsorption on SAMN surface that involves not only electrostatic interactions. In fact, at acidic $\mathrm{pH}$, we observed the highest amount of bound molecules per nanoparticle. From a purely electrostatic point of view, we should observe a repulsive interaction between RITC and SAMN surface, because both the species are protonated at low $\mathrm{pH}$.

The complexing ability of thiocyanate and isothiocyanate group toward iron(III) is well known. In order to obtain further information on the influence of the isothiocyanate group on the binding phenomenon, we prepared three different RITC

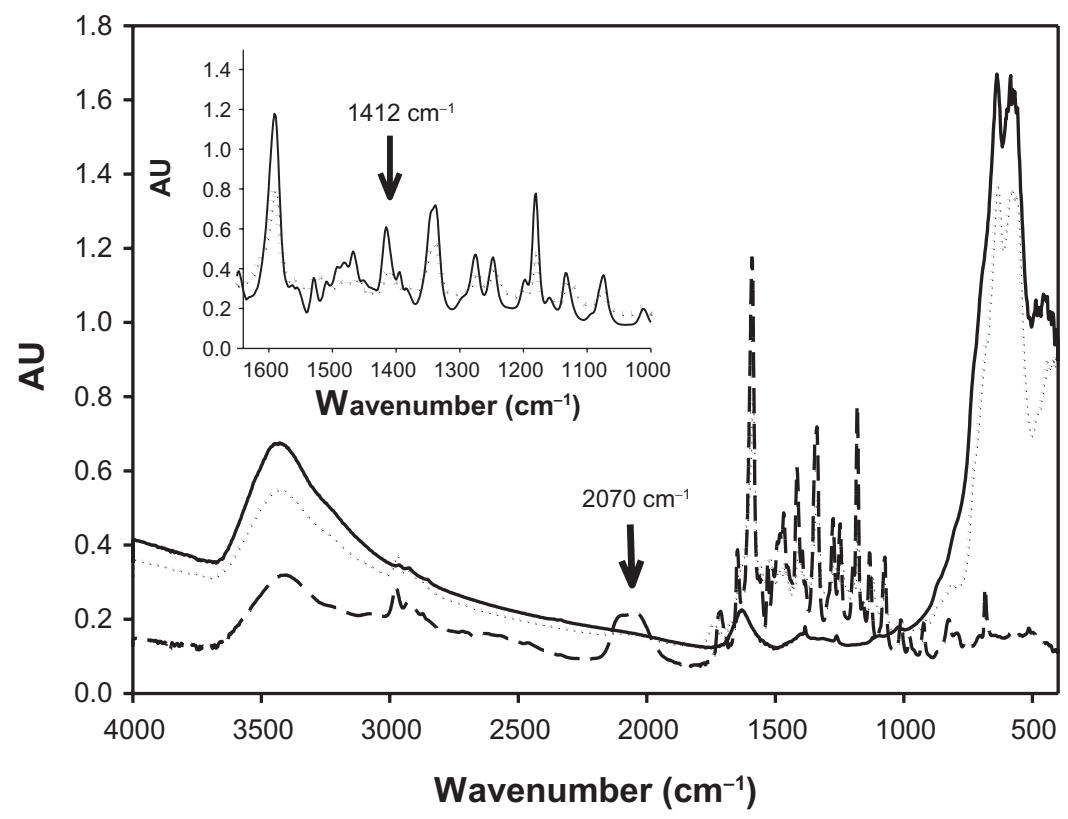

Figure 2 FTIR spectra of bare maghemite nanoparticles, rhodamine B isothiocyanate and SAMN-RITC complex. Samples were lyophilized, homogenized with KBr powder, and pelleted by an 8.0 ton hydraulic press. (-) SAMN, (---) RITC; (.......) SAMN-RITC. Inset: Comparison of FTIR spectra of RITC and SAMN-RITC complex in the region 1365-1000 cm $\mathrm{cm}^{-1}$. (-), RITC; (…...), SAMN-RITC.

Abbreviations: SAMN, surface-active maghemite nanoparticles; RITC, rhodamine B isothiocyanate; KBr, potassium bromide; FTIR, Fourier-transform infrared. 


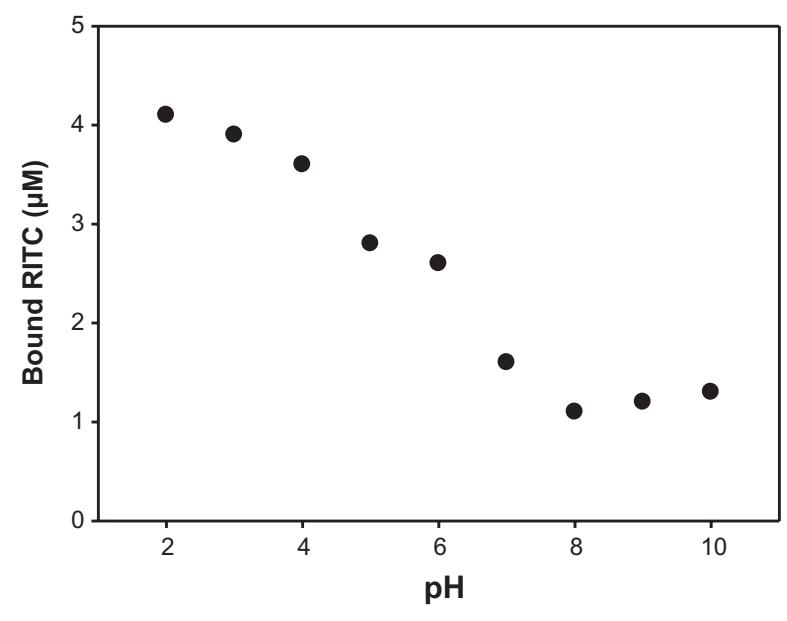

Figure 3 RITC binding to maghemite nanoparticle surface as a function of $\mathrm{pH}$. Note: Experiments were carried out in $50 \mathrm{mM}$ tetramethylammonium perchlorate in the presence of $10 \mu \mathrm{M}$ RITC and $100 \mathrm{mg} \mathrm{L}^{-1}$ SAMN, measuring the decrease of solution absorbance at $554 \mathrm{~nm}$.

Abbreviations: SAMN, surface-active maghemite nanoparticles; RITC, rhodamine B isothiocyanate.

derivatives (Table 2) formed by the condensation of different primary amines to the isothiocyanate group of the fluorescent probe, namely ethylenediamine, glycine, and 2-aminoethanol, and characterized by differently functional terminal groups. As shown in Table 2, the best binding properties were evidenced by the ethanolamine-RITC derivative, both at $\mathrm{pH} 7.0$ and at $\mathrm{pH}$ 10.0. Possibly due to the chelating properties of $-\mathrm{COOH},-\mathrm{OH}$, and $-\mathrm{NH}_{2}$, all three RITC derivatives that are used provide an anchoring function with respect to under coordinated iron sites on the nanoparticle's surface. ${ }^{37}$

It should be noted that the role of hydrophobic interactions between the RITC and nanoparticles was studied in the presence of a surfactant $(0.1 \%$ sodium dodecylsulfate $)$ and no variation of the binding process was noted and there was no evidence of hydrophobic interaction (data not shown).

\section{Surface coating of maghemite nanoparticles with RITC and bovine serum amine oxidase}

BSAO is a copper enzyme with a molar weight of approximately $170 \mathrm{kDa} .{ }^{31}$ It is a redox-protein that oxidatively deaminates polyamines containing primary amine groups (putrescine, spermidine and spermine) with the concomitant production of hydrogen peroxide, the corresponding aldehydes, and ammonia..$^{17,24}$

Preliminary experiments showed that BSAO cannot bind directly on the surface of SAMNs. Thus, in order to immobilize BSAO on SAMNs surface, RITC was used as a spacer-arm. This presents the isothiocyanate functionality
Table 2 Binding of RITC derivatives to bare maghemite nanoparticles

\begin{tabular}{|c|c|c|}
\hline \multirow[t]{3}{*}{ RITC-derivative } & \multicolumn{2}{|c|}{$\begin{array}{l}\text { Number of bound } \mu \mathrm{mol} \\
\text { RITC per } \mathrm{g} \text { nanoparticle }\end{array}$} \\
\hline & \multicolumn{2}{|c|}{ Experimental conditions } \\
\hline & $\overline{\mathrm{pH} 7.0}$ & $\mathrm{pH} I 0.0$ \\
\hline None & 2.5 & $\mathrm{I}$ \\
\hline$-\mathrm{NH}-\mathrm{CH}_{2}-\mathrm{NH}_{2}$ & 2 & 5 \\
\hline$-\mathrm{NH}-\mathrm{CH}_{2}-\mathrm{CH}_{2}-\mathrm{COOH}$ & 11 & 7 \\
\hline$-\mathrm{NH}-\mathrm{CH}_{2}-\mathrm{CH}_{2}-\mathrm{OH}$ & 17 & 12 \\
\hline
\end{tabular}

Note: The binding process was carried out in $50 \mathrm{mM}$ tetramethylammonium perchlorate in the presence of $100 \mathrm{mg} / \mathrm{L}$ maghemite nanoparticles.

to which primary amino groups of the enzyme molecule can be covalently linked.

The binding reaction was performed by adding $11 \mu \mathrm{M}$ BSAO in $0.1 \mathrm{M} \mathrm{KCl}, \mathrm{pH} 7.0$, to a suspension containing $5 \mathrm{~g}$ $\mathrm{L}^{-1}$ SAMN, which were previously covered by $29.1 \mu \mathrm{mol}$ RITC $g^{-1}$ SAMN. The solution was stirred overnight at $4^{\circ} \mathrm{C}$. The unbound enzyme was removed by repeated nanoparticle washing cycles with the aid of an external magnetic field. After three washing cycles, the activity of the immobilized enzyme was constant and no detectable enzyme release was observed in the solution. The enzyme-bound nanoparticles could be used for the preparation of magnetically recoverable fluorescent nanocatalyst.

Transmission electron micrographs show the coating of BSAO on SAMN-RITC, which forms a less electron-dense shell that covers the iron oxide nanoparticles. The average shell thickness ranges from 6-8 nm (Figure 4).

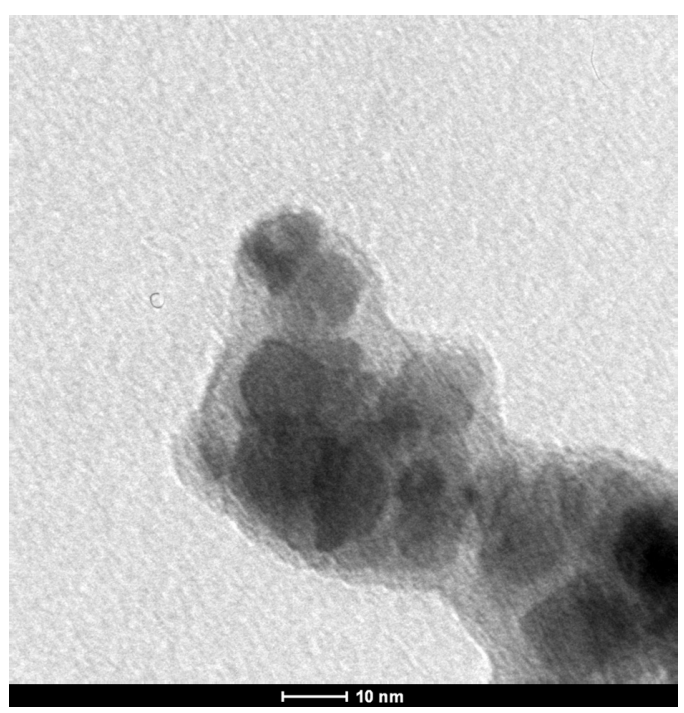

Figure 4 TEM image of maghemite nanoparticles derivatized with RITC and with immobilized BSAO (SAMN-RITC-BSAO).

Abbreviations: BSAO, bovine serum copper-containing amine oxidase; SAMN, surface-active maghemite nanoparticles; RITC, rhodamine B isothiocyanate; TEM, transmission electron microscopy. 
By comparing the trypsin digestion of native and bound enzymes, the amount of immobilized BSAO on SAMNs was determined by mass spectrometry and characterized by the same enzymatic activity, as reported in the Materials and methods section of this research. The digestion yielded $42 \%$ sequence coverage between the two samples (Figure 5). The ten most abundant common peptides, characterized by a score $>35(P<0.01)$ and accounting for about $22 \%$ of the sequence coverage, were quantitatively compared to calculate the specific activity of bound BSAO (Table 3 ). The aggregate signal of the ten peptides from maghemite-bound BSAO was 2.85 times higher than the aggregate signal of the EIC from the free protein with the same activity.

After immobilization, enzyme activity was calculated by measuring the rate of amine oxidase-catalyzed oxidation of spermine. The initial reaction rate was measured as a function of substrate concentration determining the hydrogen peroxide production in the presence of free or immobilized enzymes. Measurements of immobilized enzyme activity were performed by stirring. Under our experimental conditions, the reaction rate was independent of the stirring rate. This indicates that the kinetic of the reaction is not diffusion-controlled. Table 4 shows the kinetic parameters obtained by measuring the activity of native and immobilized BSAO. In the case of nanoparticles without rhodamine coating, no enzyme activity associated with nanoparticles was detected. The comparison of the enzymatic activity of BSAO bound to SAMN-RITC or free in solution, under the same experimental conditions, indicates that BSAO was successfully immobilized on the magnetically drivable fluorescent SAMN, displaying detectable catalytic activity on spermine $\left(\mathrm{k}_{\mathrm{c}}=14.4 \mathrm{~min}^{-1}\right)$. Immobilized BSAO shows a catalytic constant of about $30 \%$ of the value shown by native BSAO, corresponding to $0.81 \mathrm{IU} \mathrm{g}^{-1} \mathrm{SAMN}$. The value of the enzyme Michaelis constant, $\mathrm{K}_{\mathrm{M}}$, was almost doubled upon binding. Correspondingly, the binding process led to a sixfold decrease of the BSAO catalytic efficiency, $\mathrm{k}_{\mathrm{c}} / \mathrm{K}_{\mathrm{M}}$.

The system SAMN-RITC-BSAO was removed from the solution by application of an external magnet. Monitoring the disappearance of fluorescence from the assay solution and activity measurements were repeated. This shows that SAMN-RITC-BSAO maintains its catalytic activity over repeated washing cycles.

\section{Discussion}

In order to test bare maghemite surface properties, we utilized different fluorescent probes. Among the probes reported in Figure 1, only RITC was able to bind to the nanoparticle surface. The comparison of fluorophore molecule structures indicates that multiple possible interaction sites belonging to RITC could interact with the surface of maghemite

1 MFIFIFLSLW TLLVMGREEG GVGSEEGVGK QCHPSLPPRC PSRSPSDQPW

51 THPDQSQLFA DLSREELTTV MSFLTQQLGP DLVDAAQARP SDNCVFSVEL

101 QLPPKAAALA HLDRGSPPPA REALAIVFFG GQPQPNVTEL VVGPLPQPSY

151 MRDVTVERHG GPLPYYRRPV LLREYLDIDQ MIFNRELPQA AGVLHHCCSY

201 KQGGQKLLTM NSAPRGVQSG DRSTWFGIYY NITKGGPYLH PVGLELLVDH

251 KALDPADWTV OKVFFOGRYY ENLAQLEEQF EAGQVNVVVI PDDGTGGFWS

301 LKSOVPPGPT PPLOFHPOGP RFSVQGNRVA SSLWTFSFGL GAFSGPRVFD

351 VRFQGERLAY EISLOEAGAV YGGNTPAAML TRYMDSGFGM GYFATPLIRG

401 VDCPYLATYM DWHFVVESQT PKTLHDAFCV FEQNKGLPLR RHHSDFLSHY

451 FGGVAOTVLV FRSVSTMLNY DYVWDMVFYP NGAIEVKLHA TGYISSAFLF

501 GAARRYGNQV GEHTLGPVHT HSAHYKVDLD VGGLENWVWA EDMAFVPTAI

551 PWSPEHQIQR LQVTRKOLET EEOAAFPLGG ASPRYLYLAS KOSNKWGHPR

601 GYRIOTVSFA GGPMPONSPM ERAFSWGRYQ LAITQRKETE PSSSSVFNQN

651 DPWTPTVDFS DFINNETIAG KDLVAWVTAG FLHIPHAEDI PNTVTVGNGV

701 GFFLRPYNFF DQEPSMDSAD SIYFREGQDA GSCEINPLAC LPQAATCAPD

751 LPVFSHGGYP EY

Figure 5 Amino acid sequence coverage of native and bound BSAO.

Notes: Bold letters indicate amino acid total sequence coverage (42\%) obtained after trypsin digestion and analysis by MS/MS mass spectrometry of nanoparticle-bound BSAO. Underscore, bold letters indicate the amino acid sequences that were used for relative quantification of nanoparticle-bound BSAO.

Abbreviations: BSAO, bovine serum copper-containing amine oxidase; MS/MS, tandem mass spectroscopy. 
Table 3 The ten most abundant 10 common peptides obtained after trypsin digestion of native and SAMN bound BSAO

\begin{tabular}{llll}
\hline Peptide number & Score & Bound BSAO & Native BSAO \\
\hline I. YLYLASK & 36 & 747655 & 132977 \\
2. ALDPADWTVQK & 67 & 7693036 & 10761424 \\
3. GGPYLHPVGLELLVDHK & 55 & 11988500 & 17391553 \\
4. YMDSGFGMGYFATPLIR & 101 & 9442405 & 4823422 \\
5. ALDPADWTVQKVFFQGR & 44 & 1239335 & 539496 \\
6. KQLETEEQAAFPLGASPR & 87 & 13640798 & 9686214 \\
7. SQVPPGPTPPLQFHPQGPR & 63 & 113110365 & 15533939 \\
8. IQTVSFAGGPMPQNSPMER & 132 & 44140030 & 11272321 \\
9. LAYEISLQEAGAVYGGNTPAAMLTR & 169 & 3758314 & 1981715 \\
I0. HHSDFLSHYFGGVAQTVLVFR & 41 & 4856416 & 1648758 \\
Sum & & 210616854 & 73771819 \\
\hline
\end{tabular}

nanoparticles. A possible interaction with the carboxyl groups was studied by comparing the behavior of fluorescein probe in $50 \mathrm{mM}$ tetramethylammonium perchlorate, as suggested in the literature. ${ }^{38}$ At neutral $\mathrm{pH}$, the carboxyl group is negatively charged. The probe was incubated with nanoparticles as illustrated above. In this case, no binding was observed. This suggests that the probe, at $\mathrm{pH} 7.0$, did not interact with the nanoparticle surface. It can also be deduced that the carboxyl group is not involved in the binding of RITC.

The adduct formed with maghemite nanoparticles and RITC (SAMN-RITC), after washing and freeze-drying, was studied by FTIR spectroscopy. It is known that the major absorption bands of iron oxides locate in the region $400-800 \mathrm{~cm}^{-1}$ (Figure 2). In this case, the distinctive features of maghemite (bands at 445 and $630 \mathrm{~cm}^{-1}$ ) indicate cation vacancies. ${ }^{39,40}$ Furthermore, the comparison between the spectra of free RITC and of SAMN-RITC shows absorption bands at 2030-2150, $1589,1412,1345,1274,1249$, and $1180 \mathrm{~cm}^{-1}$ (Figure 2). These bands are attributable to the isothiocyanate group, the stretching vibrational mode of $\mathrm{C}=\mathrm{C}$ in benzene rings, the bending vibration of $\mathrm{CH}_{2}$ in $-\mathrm{N}-\left(\mathrm{C}_{2} \mathrm{H}_{5}\right)_{2}$, the vibration of $\mathrm{C}-\mathrm{N}$-linked benzene ring, the vibration of $\mathrm{C}-\mathrm{O}$ of $\mathrm{COOH}$, the vibration of $\mathrm{C}-\mathrm{N}$ in $-\mathrm{N}-\left(\mathrm{C}_{2} \mathrm{H}_{5}\right)_{2}$ group, and the asymmetric stretch vibration of $\mathrm{C}-\mathrm{O}-\mathrm{C}$, respectively. ${ }^{35}$ No apparent changes were found in absorption bands at 1589, 1345, 1274, 1249, and $1180 \mathrm{~cm}^{-1}$. Conversely, the relevant broadening of FTIR vibration at $1412 \mathrm{~cm}^{-1}$, attributable to $\mathrm{CH}_{2}$ bending of $-\mathrm{N}-\left(\mathrm{C}_{2} \mathrm{H}_{5}\right)_{2}$ groups (inset of Figure 2) and the disappearance of the FTIR band at

Table 4 Kinetic parameters of native and SAMN-bound BSAO

\begin{tabular}{llll}
\hline Enzyme form & \multicolumn{3}{l}{ Kinetic parameters } \\
\cline { 2 - 4 } & $\mathbf{K}_{\mathbf{M}}(\mu \mathbf{M})$ & $\mathbf{k}_{\mathrm{c}}\left(\mathbf{m i n}^{-1}\right)$ & $\mathbf{k}_{\mathrm{c}} / \mathbf{K}_{\mathbf{M}}\left(\mu \mathbf{M}^{-1} \mathbf{m i n}^{-1}\right)$ \\
\hline Native BSAO & 7.4 & 46.8 & 6.3 \\
SAMN-RITC-BSAO & 14.0 & 14.4 & 1.0 \\
\hline
\end{tabular}

Abbreviations: BSAO, bovine serum copper-containing amine oxidase; SAMN, surface-active maghemite nanoparticles; RITC, rhodamine B isothiocyanate.
$2030-2150 \mathrm{~cm}^{-1}$, is attributable to the isothiocyanate group. This suggests the involvement of these functionalities in the binding. These results suggest a multiple point binding that involves, in addition to the $-\mathrm{S}=\mathrm{C}=\mathrm{N}$ functionality, the tertiary amino groups of the RITC xanthene condensed rings. This is in contrast to the other probes tested that do not contain these two functionalities at the same time. Thus, the presence of the $-\mathrm{S}=\mathrm{C}=\mathrm{N}$ moiety on the fluorescent molecule had a positive effect on nanoparticle binding. Nevertheless, this chemical functionality is not sufficient to guarantee a stable adduct formation, as observed by incubating SAMN with fluorescein isothiocyanate. The tertiary amino groups of the RITC xanthene condensed rings, as indicated by the broadening of the FTIR band at $1412 \mathrm{~cm}^{-1}$, are not able to guarantee a stable adduct formation without the concomitant participation of isothiocyanate, as demonstrated by experiments with rhodamine B. In order to verify the involvement of the isothiocyanate group already evidenced by FTIR spectroscopy, three different amines have been bound to RITC (glycine, ethylene-diamine, and ethanolamine). Modified RITC molecules have been incubated with nanoparticles in $50 \mathrm{mM}$ tetramethylammonium perchlorate and tested at $\mathrm{pH} 7.0$ and 10.0. The amount of modified RITC molecules interacting with nanoparticles depends on the modification of the isothiocyanate group. This influences the ability of the fluorescent probe to interact with the nanoparticle's surface and confirms the role of the isothiocyanate group in the binding, even if modified as a thiorurea.

The peculiarities of RITC allow the interaction with maghemite nanoparticle surface and the formation of a stable adduct (SAMN-RITC). This magnetic nanomaterial adduct was stable across a wide $\mathrm{pH}$ range, even if the number of RITC molecules bound to the surface of SAMN was dependent on $\mathrm{pH}$.

Exploiting the chemical properties of the isothiocyanate functionality, this paper describes the surface modification of BSAO that was covalently bound to RITC to develop a 
magnetically controllable rhodamine-based nanocatalyst. The immobilization, as a monomolecular layer, allows an improved performance of the enzyme by retaining its catalytic activity towards the oxidation of spermine for more than ten days. In tumor cells, the prepared fluorescent magnetically drivable nanocatalyst could be easily used to produce cytotoxic products, hydrogen peroxide, and aldehydes, due to the high concentration of endogenous polyamines (spermine or spermidine) or, alternatively, to remove spermine and other BSAO substrates from aqueous solutions. The presence of the nanocatalyst can be conveniently monitored by its fluorescence. Moreover, the nanocatalyst can be quickly removed by the application of an external magnet and re-used without loss of the catalytic efficiency. The results demonstrate that the polyamine-degrading enzyme BSAO is a very good candidate to develop a magnetic fluorescent adduct. These results can be used in further investigations of BASO's therapeutic potential in mice-bearing tumors of various human origins. We expect that these studies will suggest new strategies to potentiate the killing of cancer cells using BSAO, which might be essential for discovering a novel anticancer therapy.

Magnetic nanomaterials are generally used as theranostic tools as drug carriers, in hyperthermia treatment, or as contrast agent in MRI. ${ }^{41,42}$

The novelty of the present approach is represented by the possibility to drive the catalytic activity of an enzyme, BSAO, with an easily magnetically removable, fluorescentdetectable nanocomposite that is able to transform enzymatic products into cytotoxic molecules in tumor cells. The advantages of this new strategy are its high specificity due to enzyme substrate accumulation in tumor cells, the ease of detectability by fluorescence imaging and targeting, and removability by magnetic field application.

Alternatively, the SAMN-RITC-BSAO adduct could be used as a magnetically drivable nanoreactor to reduce polyamine content of common foodstuffs in an attempt to reduce the polyamine intake in cancer patients. As previously shown by Bardocz et al, ${ }^{43}$ some food ingredients contain large quantities of polyamines and preliminary clinical trials performed in metastatic hormone-refractory prostate cancer patients have revealed the feasibility of a nutrition therapy based upon a 6-month polyamine-reduced $\operatorname{diet.}^{44,45}$

In conclusion, it is worth pointing out that the production process is very cheap and, by not involving organic solvents surfactants or particular capping strategies, it can be considered a "green chemistry" product. Nevertheless, the favorable magnetic properties of the material are guaranteed by optimizing the magnetic material/organic shell ratio.

\section{Acknowledgments}

This work was funded in part by Progetti di Ateneo (A.0ED00.0487PRAT08), by the University of Padua, by the Italian MIUR (Ministero dell'Istruzione, dell'Università e della Ricerca), by Istituto Superiore di Sanità "Project ItalyUSA", by Istituto Pasteur-Fondazione Cenci Bolognetti, and by funds MIUR-PRIN (Cofin). The work was supported by the Operational Program Research and Development for Innovations-European Social Fund (CZ.1.05/2.1.00/03.0058).

\section{Disclosure}

The authors report no conflicts of interest in this work.

\section{References}

1. Selvan ST, Hayakawa T, Nogami M, et al. Sol-gel derived gold nanoclusters in silica glass possessing large optical nonlinearities. J Phys Chem B. 2002;106(39):10157-10162.

2. Palecek E, Fojta M. Magnetic beads as versatile tools for electrochemical DNA and protein biosensing. Talanta. 2007;74(3):276-290.

3. Ravindranath SP, Mauer LJ, Deb-Roy C, Irudayaraj J. Biofunctionalized magnetic nanoparticle integrated mid-infrared pathogen sensor for food matrixes. Anal Chem. 2009;81(8):2840-2846.

4. Chen WJ, Tsai PJ, Chen YC. Functional nanoparticle-based proteomic strategies for characterization of pathogenic bacteria. Anal Chem. 2008;80(24):9612-9621.

5. Taylor JI, Hurst CD, Davies MJ, Sachsinger N, Bruce IJ. Application of magnetite and silica-magnetite composites to the isolation of genomic DNA. J Chromatogr A. 2000;890(1):159-166.

6. Yang HH, Zhang SQ, Chen XL, Zhuang ZX, Xu JG, Wang XR. Magnetite-containing spherical silica nanoparticles for biocatalysis and bioseparations. Anal Chem. 2004;76(5):1316-1321.

7. Ito A, Shinkai M, Honda H, Kobayashi T. Medical application of functionalized magnetic nanoparticles. J Biosci Bioeng. 2005;100(1): $1-11$.

8. Tanaka T, Matsunaga T. Fully automated chemiluminescence immunoassay of insulin using antibody-protein A-bacterial magnetic particle complexes. Anal Chem. 2000;72(15):3518-3522.

9. Neuberger T, Schopf B, Hofmann H, Hofmann M, Rechenberg BV. Superparamagnetic nanoparticles for biomedical applications: possibilities and limitations of a new drug delivery system. J Magn Magn Mater. 2005;293(1):483-496.

10. Liu Z, Liu Y, Yang H, Yang Y, Shen G, Yu R. A phenol biosensor based on immobilizing tyrosinase to modified core-shell magnetic nanoparticles supported at a carbon paste electrode. Anal Chim Acta. 2005;533(1):3-9.

11. Magro M, Valle G, Russo U, Nodari L, Vianello F, inventors; University of Padua, Magro M, Valle G, Vianello F. assignees. Maghemite nanoparticles and method for preparing thereof. European patent PCT/ EP2010/060486. July 15, 2010.

12. Magro M, Sinigaglia G, Nodari L, et al. Charge binding of rhodamine derivative to $\mathrm{OH}-[$ superscript] stabilized nanomaghemite: Universal nanocarrier for construction of magnetofluorescent biosensors. Acta Biomaterialia. 2012 Feb 13 [Epub ahead of print]. Available from: http:// dx.doi.org/10.1016/j.actbio.2012.02.005. Accessed 20 April, 2012.

13. Knowles PF, Dooley DM. Amine oxidases. In: Sigel H, Sigel A, editors. Metal Ions in Biological Systems. New York, NY: Marcel Dekker; 1994:361-403. 
14. Casero RA Jr, Pegg AE. Polyamine catabolism and disease. Biochem J. 2010;421(3):323-338.

15. Janes SM, Klinman JP. An investigation of bovine serum amine oxidase active site stoichiometry: evidence for an aminotransferase mechanism involving two carbonyl cofactors per enzyme dimer. Biochemistry. 1991;30(18):4599-4605.

16. Cai DY, Klinmann JP. Evidence for a self-catalytic mechanism of 2,4,5-trihydroxyphenylalanine quinone biogenesis in yeast copper amine oxidase. J Biol Chem. 1994;269(51):32039-32042.

17. Mondovì B, Riccio P, Agostinelli E, Marcozzi G. Oxidation of diamines and polyamines. In: Bachrach U, Heimer YM, editors. The Physiology of Polyamines. Vol 1. Boca Raton, FL: CRC Press; 1989:177-201.

18. Bachrach U. Polyamines as indicators of disease activity and response to therapy in cancer patients. In: Bachrach U, Heimer YM, editors. The Physiology of Polyamines. Vol 2. Boca Raton, FL: CRC Press; 1989:234-249.

19. Agostinelli E, Tempera G, Molinari A, et al. The physiological role of biogenic amines redox reactions in mitochondria. New perspective in cancer therapy. Amino Acids. 2007;33(2):175-187.

20. Lindsay GS, Wallace HM. Changes in polyamine catabolism in HL-60 human promyelogenous leukaemic cells in response to etoposideinduced apoptosis. Biochem J. 1999;337(Pt 1):83-87.

21. Calcabrini A, Arancia G, Marra M, et al. Enzymatic oxidation product of spermine induce greater cytotoxic effects on human multidrug-resistant colon carcinoma cells (LoVo) than on their wild-type counterparts. Int J Cancer. 2002;99(1):43-52.

22. Averill-Bates DA, Cherif A, Agostinelli E, Tanel A, Fortier G. Anti-tumoral effect of native and immobilized bovine serum amine oxidase in a mouse melanoma model. Biochem Pharmacol. 2005;69(12):1693-1704.

23. Agostinelli E, Seiler N. Non-irradiation-derived reactive oxygen species (ROS) and cancer. Therapeutic implications. Amino Acids. 2006;31(3):341-355.

24. Agostinelli E, Belli F, Molinari A, et al. Toxicity of enzymatic oxidation products of spermine to human melanoma cells (M14): sensitization by heat and MDL 72527. Biochim Biophys Acta. 2006;1763(10):1040-1050.

25. Agostinelli E, Belli F, Dalla Vedova L, Marra M, Crateri P, Arancia G. Hyperthermia enhances cytotoxicity of amine oxidase and spermine on drug-resistant colon adenocarcinoma cells (LoVo). Int J Oncol. 2006;28(6):1543-1553.

26. Agostinelli E, Dalla Vedova L, Belli F, Condello M, Arancia G, Seiler N. Sensitization of human colon adenocarcinoma cells (LoVo) to reactive oxygen species by lysosomotropic compounds. Int $J$ Oncol. 2006;29(4):947-955.

27. Agostinelli E, Arancia G, Dalla Vedova L, et al. The biological functions of polyamine oxidation products by amine oxidases: perspectives of clinical applications. Amino Acids. 2004;27(3-4):347-358.

28. Turini P, Sabatini S, Befani O, et al. Purification of serum amine oxidase. Anal Biochem. 1982;125(2):294-298.

29. Stevanato R, Mondovì B, Sabatini S, Rigo A. Spectrophotometric assay for total polyamines by immobilized amine oxidases. Anal Chim Acta. 1990;237:391-397.
30. Bradford MM. A rapid and sensitive method for the quantitation of microgram quantities of protein utilizing the principle of protein-dye binding. Anal Biochem. 1976;72:248-254.

31. Bellelli A, Morpurgo L, Mondovì B, Agostinelli E. The oxidation and reduction reactions of bovine serum amine oxidase. a kinetic study. Eur J Biochem. 2000;267(11):3264-3269.

32. Lucas T, Dubois E, Chevalet J, Durand-Vidal S, Joiret S. Reactivity of nanocolloidal particles $\gamma-\mathrm{Fe}_{2} \mathrm{O}_{3}$ at charged interfaces. Part 2 . Electrochemical conversion. Role of the electrode material. Phys Chem Chem Phys. 2008;10(22):3274-3286.

33. Valeur B. Molecular Fluorescence: Principles and Applications. Weinheim, Germany: Wiley-VHC; 2002.

34. Hung CW, Holoman TRP, Kofinas P, Bentley WE. Towards oriented assembly of proteins onto magnetic nanoparticles. Biochem Eng J. 2008;38:164-170.

35. Liu X, Cui D, Wang Q, Xu H, Li M. Photoluminescence enhancement of ZrO2/rhodamine B nanocomposite. J Mat Sci. 2005;40(5):1111-1114.

36. Vieira Ferreira LF, Cabral PV, Almeida P, Oliveira AS, Reis MJ, Botelho do Rego AM. Ultraviolet/visible absorption, luminescence, and X-ray photoelectron spectroscopic studies of a rhodamine dye covalently bound to microcrystalline cellulose. Macromolecules. 1998;31(12):3936-3944.

37. Chen LX, Liu, T, Thurnauer MC, Csencsits R, Rajh T. $\mathrm{Fe}_{2} \mathrm{O}_{3}$ nanoparticle structures investigated by $\mathrm{X}$-ray absorption near-edge structure, surface modifications, and model calculations. J Phys Chem B. 2002;106(34):8539-8546.

38. Pirillo S, Cornaglia L, Ferreira ML, Rueda EH. Removal of fluorescein using different oxides as adsorbents: effect of $\mathrm{pH}$. Spectrochim Acta A. 2008;71(2):636-643.

39. Roca AG, Marco JF, Morales MP, Serna CJ. Effect of nature and particle size on properties of uniform magnetite and maghemite nanoparticles. J Phys Chem. 2007;111(5):18577-18584.

40. Daou TJ, Greneche JM, Pourroy G, et al. Coupling agent effect on magnetic properties of functionalized magnetite-based nanoparticles. Chem Mater. 2008;20(18):5869-5875.

41. Kelkar SS, Reineke TM. Theranostics: combining imaging and therapy. Bioconjug Chem. 2011;22(10):1879-1903.

42. Duncan R, Gaspar R. Nanomedicine(s) under the microscope. Mol Pharm. 2011;8(6):2101-2141.

43. Bardocz S, Duguid TJ, Brown DS, et al. The importance of dietary polyamines in cell regeneration and growth. Br J Nutr. 1995;73(6):819-828.

44. Cipolla B, Guillè F, Moulinoux JP. Polyamine-reduced diet in metastatic hormone-refractory prostate cancer patients. Biochem Soc Trans. 2003;31(2):384-387.

45. Cipolla BG, Havouis R, Moulinoux JP. Polyamine contents in current foods: a basis for polyamine reduced diet and a study of its long term observance and tolerance in prostate carcinoma patients. Amino Acids. 2007;33(2):203-212.
International Journal of Nanomedicine

\section{Publish your work in this journal}

The International Journal of Nanomedicine is an international, peerreviewed journal focusing on the application of nanotechnology in diagnostics, therapeutics, and drug delivery systems throughout the biomedical field. This journal is indexed on PubMed Central,

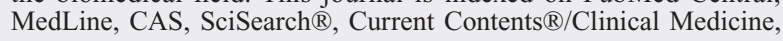

\section{Dovepress}

Journal Citation Reports/Science Edition, EMBase, Scopus and the Elsevier Bibliographic databases. The manuscript management system is completely online and includes a very quick and fair peer-review system, which is all easy to use. Visit http://www.dovepress.com/ testimonials.php to read real quotes from published authors. 